\title{
Deanthropomorphized Pancomputationalism and the Concept of Computing
}

\author{
Paweł Polak ${ }^{1}$, Roman Krzanowski ${ }^{2}$
}

\begin{abstract}
Pancomputationalism is quite a wide-ranging concept, but most of its variants, either implicitly or explicitly, rely on Turing's conceptualizations of a computer and computing, which are obvious anthropomorphisms. This paper questions the concept of pancomputationalism based on Turing computing and asks what concept of computation can be used to avoid the constrains of anthropomorphisations.
\end{abstract}

Keywords: pancomputationalims, Turing machines, computer, antropomorphisation, computing.

\section{Introduction}

Computer science since its birth use a concept of computing which is based on the concept of Turing computation $[35]^{3}$. The Turing computation model while very successful has its limitations [36]. These limitations are seen specifically in the modeling of nature (see e.g. [33]). This results in an interesting paradox: on one hand we claim that nature is computational in Turing sense $[2,30]$, on the other hand certain natural processes are beyond the computing capacity/capability of the Turing model (see e.g. [9, 34]). If we assume that nature in some way computes better than Turing model does, abandoning the Turing computational model as the sole model of computation would open to us a new possibilities, closed by sticking to the Turing model ${ }^{4}$. Thus, investigating natural computing denoted often interpretations as pancomputationalism may change for the better the way we see and practice computing.

1 Pontifical University of John Paul II in Kraków, Faculty of Philosophy, Chair of History and Philosophy of Science, 31-002 Kraków, ul. Kanonicza 9; e-mail: <atpolakp@cyf-kr.edu.pl>.

2 Pontifical University of John Paul II in Kraków, Faculty of Philosophy, 31-002 Kraków, ul. Kanonicza 9.

3 We use the terms "Turing computation", "Turing computing", "Turing concept of computing", "Turing machine", and "Turing model" as synonyms, which we admit is not entirely correct. But in the context of the paper we judge this use of different terms to be acceptable and not leading to the misunderstandings and obfuscation of the discussed issues.

4 This view was inspired by Peter Denning claims that considering natural computational processes is the best way to develop the computing as a discipline [11]. In his view "computing is evolving constantly." Consequently he stated: "Computing is no longer a science of just the artificial. It is the study of information processes, natural and artificial." 
Ontic pancomputationalism (later referred to as pancomputationalism) is the notion that nature, or the universe, is a computer, and what nature does is essentially computation [4, 16, 38-40]. There are many versions of pancomputationalism depending on how one attribute computational powers to nature- everything computes, some processes compute, or only special processes compute (see e.g. [30]). Only digital pancomputationalism explicitly claims that the universe is the Turing machine (TM) (see e.g. [4]). But other versions of pancomputationalism (unlimited, limited, ontic, strong, weak, limited, causal to name just a few, see. e.g. [2, 30]) implicitly rely on Turing's concepts of computing assuming algorithmic procedure $[31]^{5}$. Thus, we may safely state that pancomputationalism is therefore de facto Turing pancomputationalism [3, 27, 30].

Is it bad, indifferent or beneficial to use Turing concept of computing as the model of the universe? Of course, there is no definite answer to this question. However, we may speculate that with known limitations of Turing computing disclosed by the concept of hypercoputation $[6,7,36]$, with the postulate that the universe is Turing-like computing system we import limitations of the Turing computing model (and our understanding of) onto the Universe, which obviously would not be an advisable move. To restate the argument, if the Turing model of computation brings important limitations (more on this topic see [32]), imposing it on the Universe gives us the limited picture of the universe, preventing us from seeing it as it may be.

We therefore pose the question of how pancomputationalism can, or should, be reinterpreted if we were to free ourselves from our dependency on Turing's conceptualization of computing?

It is also important to realize that the idea of Turing computing and Turing machines are strongly based on analogies to human kind. Indeed, the Turing machine is modeled after an idealized mechanized clerk (see e.g. [5]). Thus, in pancomputaionalism, rejecting Turing's model of computation, we would be in fact deanthropomorphizing it. We all know (the claim hardly needs further justification) that any antropomorphizations in science lead to dead ends, if not to outright embarrassments [1, 10]. Thus, "deanthropomorphizing pancomputationalism", if we agree that Turing computing is the factor centered around human person (like the Ptolemaic model of the universe), should open for us new perspectives on computing, computer and the Universe, as the deanthropomorphizing of the Universe did to the concept of Cosmos [22] (see section 4).

Thus, we ask again would this deanthropomorphization of pancomputationalism open up a new perspective onto pancomputaionalizm? And may be, would the deanthropomorphized concept of pancomputationalism lead to a redefinition of our concept of computers and computing?

\section{What does pancomputationalism assume?}

We should focus on the three main assumptions behind pancomputationalism $[4,14,39]:^{6}$

5 There are of course some exceptions, e.g. Witold Marciszewski's view on pancomputationalism. It is founded on the Turing concept of computing, however it is enriched by assumption of the possibility of infinite computation in reality on data with infinite description [25].

6 While looking at different formulations of ontic pancomputationalism, we could note the striking fact that almost all of them assume digital ontology and some form of digital computation (i.e., a Turing machine or universal cellular automaton). 
1. Digitalism, the claim that the physical world is a digital structure;

2. Physical pancomputationalism, the claim that physical objects perform computational processes; and

3. Zuse's thesis, the claim that the physical world is a universal computer.

The first assumption of digitalism requires a very heavy ontological commitment. In its stronger version, it claims that "the physical world is isomorphic to a digital structure." In its weaker version, it claims that "the physical world is isomorphic to a mathematical structure." These assumptions and their consequences have been subjected to detailed analysis [4].

The most important argument in support of digitalism comes from fundamental physics. According to current views, physical theories describing the fundamental levels of the physical world are discrete (e.g., quantum mechanics), but this argument is weak because it is not clear which properties of the universe that we currently assume to be fundamental actually constitute the fundamental level of physical reality. We cannot assume digital ontology for theories such as unification based on non-commutative geometries [18, 19].The above mentioned theory shows that the digital structures of QM and continuous structures of general relativity are only the limiting cases of more general mathematical structures.

The second argument in support of digitalism derives from the successes of discrete mathematics in modern science. However, these successes only show that natural structures can be approximated by digital structures in digital computers, and they do not support any deeper metaphysical claims about nature. ${ }^{7}$ There is no proof that these digital and natural structures are identical. Thus, while digitalism is frequently assumed by some physical theories, it cannot be defended as the ontology of reality. (For another critique of digital ontology, see [14] and [29].)

The second assumption of physical pancomputationalism, meanwhile, is very open to interpretation (e.g. [29]), so in its generic form, without additional qualification about what computation means, it does not contribute much to the overall concept of pancomputationalism. It can therefore be simply omitted without any consequences for the definition.

It is also difficult to support Zuse's thesis rejecting digitalism. Zuse's concept of computation is strongly tied to Turing's concept, making his thesis strongly anthropomorphic. A thorough analysis of Zuse's model can be found in the work of Beraldo-de-Araújo and Baravalle [4].

\section{More on Zuse's thesis}

Zuse claims that the universe is a universal computer, but an uncritical acceptance of this thesis could lead to " $a$ danger for very general ideologies that seem to explain everything" [13 pp. 25-26]. According to these authors, it is safer to select a weaker thesis called realist

7 An argument concerning the possibility of assuming digital ontology was put forward by Zuse [39] and used more recently by Beraldo-de-Araújo and Baravalle [4]. 
weak pancomputationalism: "All processes can be described as computational processes because this happens to be a useful way of describing them in scientific theory." However, the statement "this happens to be a useful way" will not withstand a sustained critique, because trying to explain metaphysics through pragmatic arguments never succeeds. Thus, even the weaker version of Zuse's thesis seems unacceptable.

However, replacing the concept of a computer in Zuse's thesis with some nonanthropomorphous concept of computation is a challenge. Some possible solutions have been investigated $[14,15]$.

Zuse's thesis also brings up the issues of computational language and symbolic representation. It is not clear how we could interpret these in the context of pancomputationalism. For example, some arguments assert that natural computation ${ }^{8}$ could be better characterized by sub-symbolic computation [12]. If we need some form of instruction, we need a finite number of instructions to develop an effective description. Therefore, in some cases of non-Turing computation (see e.g. [7]), this could be satisfied. In these cases, we could (although maybe not even theoretically) obtain a functional analogy of a digital computer but without the constraints of Turing computability.

As we said above, interpretations of Zuse's thesis are strongly anthropomorphic because they rely on Turing's concept of computation and computers. We may therefore question how this aspect of Zuse's thesis affects the overall concept of pancomputationalism.

Note: In conceptualizing natural computations, we cannot assume only the mechanical (it mean algorithmic) procedure envisioned by Turing, so the classical concept of an algorithm will not suffice here. Therefore, what concept of an effective procedure could be used instead? The answer to this clearly depends on the understanding of computation.

As we stated earlier, we cannot be sure if any computational model is ultimate in the sense that it cannot be developed further, because its mathematical structure already fits perfectly to that of nature. ${ }^{9}$ It therefore seems that from a theoretical point of view (assuming pancomputationalism), the concept of computation needs to be endlessly developed, together with the concept of a computer (cf. [11]). One may ask why, though? Computation existed prior to the creation of computing devices, which are artifacts that were developed to facilitate this process. The precedence of computation over the computer seems obvious, even from just reading Turing's papers and not searching out other historical records.

\section{Anthropomorphic assumptions}

Anthropomorphism is in some sense an attribution of human characteristics to nature or animals. In the current discussion of anthropomorphization in computer sciences and informatics, anthropomorphism refers specifically to the use of human analogues in models for computation and computers.

Anthropomorphism in science has been slowly weeded out (see for example the classical article [1]) because it strongly constrains scientific theories despite the occasional

8 "Natural computation" means here the interpretation of process in nature as in some sense computational processes.

9 The close view was presented by Floridi [14], who uses a concept of "modes of presentation" of being. 
positive contributions (e.g. [23]). Modern science has found the rejection of anthropomorphism in science to be a sound approach and not open to argument.

If pancomputationalism were to follow the lead of modern science, it would have to exclude all the anthropomorphic assumptions behind pancomputationalism. ${ }^{10}$ Thus, we would not be able to a priori assume that the (mathematical) structures of nature ${ }^{11}$ are isomorphic to the mathematical structures described by our mathematics.

Drawing lines in the sand could provide inspiration for thinking about continuity, and historically, it was one of the roots leading to the mathematical concept of continuity. However, the mathematical concept is not a simple idealization or abstraction of human action. On the other hand, the mechanical procedure described by Turing (see e.g. [37 p. 436]) is precisely an idealization of human calculation. Not only was it inspired by this - it is in fact the very essence of this action (see e.g. [7 p. 697]). In the first case, anthropomorphism plays an anticipatory role, but in the second, we are dealing with a formalization of human behavior. While Turing's concept is extremely useful in science, we need to remember that it is merely a successful approximation of nature's mathematical forms. ${ }^{12}$

\section{Pancomputationalism without Turing}

The approximate nature of scientific theories leads to a question: Could we use non anthropomorphous computational processes for hypercomputation. ${ }^{13}$ It seems reasonable to use the well-defined Turing model as the base for further developing concepts of hypercomputation, but this strategy could be misleading because it includes some anthropomorphic assumptions.

So, is the concept of non anthropomorphous computation even conceivable? Theoretical physics may help here by suggesting some ways of thinking about not-anthropomorphiccomputing-nature. Regardless, we know how to use some non-computational values (in the Turing sense) as an input for physical processes. For example, nature can give us a series of random values that can be used in hardware random value generators.

We know that for non anthropomorphic computation we need to formulate a new conceptual framework, because modern concepts of computing are based on anthropomorphic assumptions. For example, excluding digital ontology for its anthropomorphous basis leads to the concept of continuous representations, but simply replacing a digital version with a continuous one may not suffice due to the need for a

10 Mycka [28 p. 257], for example, suggested that the newest physical theories show possibilities for computational systems with infinite resources. For him, this could be an argument for rejecting the human ideal of computing as a model of computation, so it is therefore an argument for replacing digital anthropomorphous computation with a nonanthropomorphous analog version.

11 We are talking here about the mathematical structures of nature as conceptualized by Michael Heller [20] (see also [24]. Similar concept see e.g. [17].

12 The anthropomorphisms in Turing's model could also play a heuristic role (anticipatory anthropomorphisms). One attempt to change the role of the anthropomorphisms in the concept of computation is the attempt to formulate the theory of Real Recursive Functions (which is described further in this section).

13 From the pancomputationalist point of view, we are using a tiny subset of the computational power of nature by applying Turing's model of computation to physical systems. All computer engineers know how to apply Turing's model to transistor-based electronic systems, but they also realize how redundant these computations are. By imposing Turing's model on the nature we are also excluding the hypercomputations. 
deeper reconstruction of the conceptual framework (which will be discussed in the following section).

We should remember that the basis of non-anthropomorphous computation has already been conceptualized [26]. Moore proposed a model of idealized computation in continuous time that shows some possibilities for the construction of non anthropomorphous computing systems. In the last decade, further theoretical development has taken place, such as the generalized theory of Real Recursive Functions [8]. Such theories promise a new paradigm in computing.

This is arguably not the final step in the development of new concepts for computation, however. For ontic pancomputationalism, only nature itself can define the target, but for the concept of a computer, there are some additional constraints.

\section{The problem of encoding}

One of the problems we face is a problem of decoding and encoding that is seen as the essence of computing. Without encoding or decoding we would trivialize the concept of computing. There could be no difference between physical process and computing.

To clarify this problem let us look at the formal definition of computing and computer formulated by Beraldo-de-Araújio et al. [4]. Computation can be formally represented by the following two definitions:

Definition 1: A process is a function $P: I \rightarrow O$ such that its domain $I$ is a set whose elements are called inputs and its co domain $\mathrm{O}$ is a set whose elements are called outputs, while both $\mathrm{I}$ and $\mathrm{O}$ are subsets of a physical world.

Definition 2: A computer is a function $C: I \rightarrow O$ from a set of input symbols $I$ to a set of output symbols $O$ such that $C(\bar{x})$ is generated from $\bar{x}$ via a computation. A process $P: I \rightarrow O$ is computational if $P$ is generated by a computer $C$, i.e., $C(\bar{x})=P(x)$ for all $x \in I$, where $\bar{x}$ is a symbolic representation of $x$.

According to Beraldo-de-Araújio et al, the essence of computation is "symbolic manipulation" and the computer is mapping function between two sets of symbols. This property of computing is also claimed by Horsman et al. [21 p. 15]. They claim that what is most important for computing processes is the possibility of encoding and decoding:

Without encode and decode steps, there is no computation; there is simply a physical system undergoing evolution. This, then, is one of the key ways in which this framework distinguishes between a physical system 'going about its business', and the same physical system undergoing the same physical evolution, but this time being used to compute. This is how we can escape from falling into the trap of "everything is information" or "the universe is a computer": a system may potentially be a computer, but without an encoding and a decoding step it is just a physical system. 
Horsman et al. show that the relationship between encoded structures is essential to the encoding/decoding process. This then translates into the notion that our theoretical structures describing the world should be isomorphic to nature's own structures. Considering ontology, our theoretical descriptions will always be approximations if ontology is limited only to the ontological commitment of accepted theories. Thus, the encoding/decoding process depends on having an appropriate theoretical description. For non anthropomorphic computations, we would not know how to achieve this. ${ }^{14}$

\section{Conclusions}

We argue in this paper that to overcome limitations of the concept of computing based on the Turing model we need to look somewhere else. One source of potential inspiration for the new conceptualization of computing is nature or natural computations.

Our current conceptualization of natural computations is denoted as pancomputationalism. Most of its variants, either implicitly or explicitly, rely on Turing's conceptualizations of a computer and computing, bringing with it, its the limitations and conceptual framework. One of the obvious problems with the Turing model (we argue) is its anthropomorphic roots. And antropomorphisation, while seductive, always puts limits on the associated concepts.

Thus, considering the current conceptualizations of pancomputationalism as strongly informed by the Turing model, we argue that a new concept of computers and computing while inspired by nature, should not be based on the anthropomorphic framework of the Turing model. That is what we call as "deanthropomorphisation of pancomputationalism".

We do not have or propose the solution to the problem of computation without Turing. However, we hope that the discussion of the limitation of the Turing-based models of pancomputationalism will encourage the search for the new, nature-inspired concepts of computing.

\section{Acknowledgments}

We would like to thank Paweł Stacewicz for discussions and some bibliographical help.

14 A pessimistic vision of the prospects of modeling of natural computations was described by Mycka [28 pp. 257-258], who stated that in practice, the problem of the boundaries of decidability in analogous computation is relative to accepted physical theories. He also suggested that there probably were some aspects of nature that could not be analyzed by humans, so the process could only be simulated rather than modeled. 


\section{References}

[1] Agassi, J., Anthropomorphism in Science. In: Dictionary of the History of Ideas: Studies of Selected Pivotal Ideas (Editor: P. P. Wiener). New York: Scribner, 1968, 97-91.

[2] Anderson, N.G., Piccinini, G., Pancomputationalism and the Computational Description of Physical Systems [preprint]. 2017.

[3] Barrow, J.D., A New Mathematics for a New Era [Matematyka nowej ery]. Philosophical Problems in Science (Zagadnienia Filozoficzne w Nauce), (16), 1994, 87-99.

[4] Beraldo-de-Araújo, A., Baravalle, L., The Ontology of Digital Physics. Erkenn, 82 (6), 2017, 1211-1231.

[5] Blass, A., Gurevich, Y., Algorithms: A quest for absolute definitions. Bulletin of the European Association for Theoretical Computer Science, 81, 2003, 195-225.

[6] Copeland, B.J., What Is Computation? Synthese, 108 (3), 1996, 335359.

[7] Copeland, B.J., The Broad Conception of Computation. American Behavioral Scientist, 40 (6), 1997, 690-716.

[8] Costa, J.F. et al., A foundation for real recursive function theory. Annals of Pure and Applied Logic, 160 (3), 2009, 255-288.

[9] Cubitt, T. et al., Undecidability of the Spectral Gap. Nature, 528 (7581), 2015, 207-211.

[10] Davies, J., Anthropomorphism in science. EMBO reports, 11 (10), 2010, 721-721.

[11] Denning, P.J., Computing is a Natural Science. Communications of the $A C M, \mathbf{5 0}$ (7), 2007, 13-18.

[12] Dodig-Crnkovic, G., The Development of Models of Computation with Advances in Technology and Natural Sciences. In: Proceedings of The 6th AISB Symposium on Computing and Philosophy: The Scandal of Computation - What is Computation? (Editors: M. Bishop and Y. J. Erden). 2013, 1-8.

[13] Dodig-Crnkovic, G., Müller, V.C., A Dialogue Concerning Two World Systems: Info-Computational vs. Mechanistic. In: Information and Computation (Editors: M. Burgin and G. Dodig-Crnkovic). Singapore: World Scientific Publishing Co., 2011, 149-184.

[14] Floridi, L., Against digital ontology. Synthese, 168 (1), 2009, 151-178. 
[15] Floridi, L., A defence of informational structural realism. Synthese, 161 (2), 2008, 219-253.

[16] Fredkin, E., An introduction to digital philosophy. International Journal of Theoretical Physics, 42 (2), 2003, 189-247.

[17] French, S., Ladyman, J., In Defence of Ontic Structural Realism. In: Scientific Structuralism (Editors: A. Bokulich and P. Bokulich). Dordrecht: Springer Netherlands, 2011, 25-42.

[18] Heller, M. et al., Noncommutative Unification of General Relativity and Quantum Mechanics. Journal of Mathematical Physics, 46 (12), 2005, 122501.

[19] Heller, M., Sasin, W., Noncommutative Unification of General Relativity and Quantum Mechanics. International Journal of Theoretical Physics, 38 (6), 1999, 1619-1642.

[20] Heller, M., Dispute around sructural realism [Spór o realizm strukturalistyczny]. In: Filozofia i wszechświat: wybór pism. Kraków: TAiWPN UNIVERSITAS, 2006, 215-234.

[21] Horsman, C. et al., When does a physical system compute? Proceedings of the Royal Society A: Mathematical, Physical and Engineering Sciences, 470 (2169), 2014, 20140182-20140182.

[22] Koyré, A., From the closed world to the infinite universe. Charleston, S.C.: Forgotten Books, 2008.

[23] Kracher, A., Imposing Order-The Varieties of Anthropomorphism. Studies in Science and Theology, 8, 2002, 239-261.

[24] Krzanowski, R., Minimal Information Structural Realism. Philosophical Problems in Science (Zagadnienia Filozoficzne w Nauce), (63), 2017, 59-75.

[25] Marciszewski, W., Universe as a computer and eschata [Wszechświat jako komputer i sprawy ostateczne]. Computerworld, (9), 1999.

[26] Moore, C., Recursion theory on the reals and continuous-time computation. Theoretical Computer Science, 162 (1), 1996, $23-44$.

[27] Müller, V.C., Pancomputationalism: Theory or Metaphor?. In: Philosophy, computing and information science (Editors: R. Hagengruber and U. Riss). London: Pickering \& Chattoo, 2014, $213-$ 221.

[28] Mycka, J., Continuous and discrete computation as an anthropomorphous and a physical concept of effective computation [Obliczenia dyskretne i ciagłe jako realizacja antropomorficznej $i$ fizycznej koncepcji efektywnej obliczalności]. In: Światy matematyki: tworzenie czy odkrywanie? Księga pamiątkowa ofiarowana 
profesorowi Romanowi Murawskiemu (Editors: I. BondeckaKrzykowska and J. Pogonowski). Poznań: Wydawnictwo Naukowe Uniwersytetu im. Adama Mickiewicza, 2010, 247-260.

[29] Pexton, M., Emergence and interacting hierarchies in shock physics. Euro Jnl Phil Sci, 6 (1), 2015, 91-122.

[30] Piccinini, G., Computation in Physical Systems. In: The Stanford Encyclopedia of Philosophy (Editor: E. N. Zalta). Metaphysics Research Lab, Stanford University, 2017.

[31] Piccinini, G., Physical computation: a mechanistic account. 2015.

[32] Piesko, M., Uncalculable calculability [Nieobliczalna obliczalność]. Kraków: Copernicus Center Press, 2011.

[33] Pour-El, M.B., Richards, J.I., Computability in Analysis and Physics. Cambridge: Cambridge University Press, 2016.

[34] Ringel, Z., Kovrizhin, D.L., Quantized gravitational responses, the sign problem, and quantum complexity. Science Advances, 3 (9), 2017, e1701758.

[35] Turing, A.M., On Computable Numbers, with an Application to the Entscheidungsproblem. Proceedings of the London Mathematical Society, s2-42 (1), 1937, 230-265.

[36] Turing, A.M., Systems of Logic Based on Ordinals. Proceedings of the London Mathematical Society, s2-45 (1), 1939, 161-228.

[37] Turing, A.M., Computing Machinery and Intelligence. Mind, 59 (236), 1950, 433-460.

[38] Wolfram, S., A new kind of science. Champaign, IL: Wolfram Media, 2002.

[39] Zuse, K., Calculating Space [Rechnender Raum]. Elektronische Datenverarbeitung, 8, 1967, 336-344.

[40] Zenil, H. ed., A computable universe: understanding and exploring nature as computation. Singapore: World Scientific, 2013. 